\title{
A new subclade of haplontic Metschnikowia species associated with insects of morning glory flowers in Africa and description of Metschnikowia aberdeeniae sp. nov.
}

\author{
Marc-André Lachance, ${ }^{1}$ T. Michael Anderson ${ }^{2}$ and William T. Starmer ${ }^{2}$ \\ ${ }^{1}$ Deparment of Biology, University of Western Ontario, London, Ontario N6A 5B7, Canada \\ ${ }^{2}$ Department of Biology, Syracuse University, Syracuse, NY 32144, USA
}

\begin{abstract}
The novel species Metschnikowia aberdeeniae is described to accommodate five isolates recovered from insects of morning glory flowers in the Serengeti National Park, Tanzania. On the basis of rDNA ITS and D1/D2 large-subunit sequences, these yeasts form, together with six other isolates, a novel subclade of large-spored Metschnikowia species. The exact position of the subclade within the Metschnikowiaceae cannot be determined with any confidence from these sequences or from small-subunit rDNA sequences, as the variable sites of the sequences are excessively divergent. However, in morphological and physiological terms, the novel isolates are typical of the genus Metschnikowia in general and of the large-spored group in particular. The type strain of Metschnikowia aberdeeniae sp. nov. is strain SUB 05-213.1 ${ }^{\top}\left(=\mathrm{CBS} 10289^{\top}=\mathrm{NRRL}\right.$ Y-27921 $)\left(h^{-}\right)$and the allotype is strain SUB 05-213.2 $\left(=\right.$CBS $10290=$ NRRL Y-27922) $\left(h^{+}\right)$.
\end{abstract}

Nitidulid beetles and other insects that visit ephemeral flowers harbour a highly specific yeast biota that includes, among others, a group of haplontic, heterothallic Metschnikowia species that form unusually large asci (Lachance et al., 2001). These species are unusual also in that their rDNA sequence divergence is nearly as extensive as that found across the entire ascomycetous yeast domain. This is in sharp contrast with the high degree of homogeneity in terms of morphology, life cycle and nutritional attributes of the species. These yeasts show distinct biogeography. Some species are restricted in distribution to the Nearctic region, Central America, Brazil, Hawaii or the Australia-South Pacific region (e.g. Lachance et al., 2005). The distribution of the related asexual species Candida ipomoeae is more or less Neotropical. Its range extends from Brazil to Tennessee but does not include the Great Lakes region. The sampling range was recently extended to the African continent. Eight sites in or near the Serengeti National Park, Tanzania, featured stands of Ipomoea hildebrandtii (Convolvulaceae), an equatorial African native morning glory. Among the yeasts recovered from insects collected in the flowers were 11 isolates that constitute a novel subclade of the genus Metschnikowia. The yeasts are typical of others recovered in similar habitats, but their sequence divergence would suggest a distant ancestry within the Metschnikowiaceae. One taxon, represented by multiple strains of both mating

The GenBank/EMBL/DDBJ accession number for the sequence of the rDNA segment encompassing most of the small subunit and most of the large subunit of strain SUB 05-213.1 ${ }^{\top}$ is DQ266429. types, is described as a novel species of the genus Metschnikowia, for which the name Metschnikowia aberdeeniae sp. nov. is proposed.

\section{Isolation, characterization and ecology}

The locations of the collection sites are detailed in Fig. 1 and Table 1. A total of 36 insects, collected in 2004 and 2005, were allowed to walk on the surfaces of plates of YM agar supplemented with $100 \mathrm{mg}$ chloramphenicol $\mathrm{l}^{-1}$. Three different insects were sampled (Table 2). The blister beetle Mylabris tristigma (Coleoptera: Meloidae) is a pollen and flower feeder regarded as an important pest of plant crops in sub-Saharan Africa (Abate \& Ampofo, 1996). An unidentified buprestid beetle (also known to cause flower damage) and an unidentified Scaptodrosophila species also yielded yeasts. Five of the eight collection sites yielded isolates that were kept for further analysis. The yeasts were characterized morphologically and physiologically by using standard methods (Yarrow, 1998). At least one representative of each morphotype of ascomycetous yeast was also identified by PCR amplification and rDNA sequencing, as described previously (Lachance et al., 2005). Mating reactions among Metschnikowia strains were obtained by mixing actively growing cells in pairs on yeast carbon base agar supplemented with $0.01 \%$ ammonium sulphate. Asci were detected by scanning the plates directly at low magnification after 1 day. The presence of ascospores was assessed after 2-3 days in material mounted on a slide, at higher magnifications. Strains were accessioned in the culture 


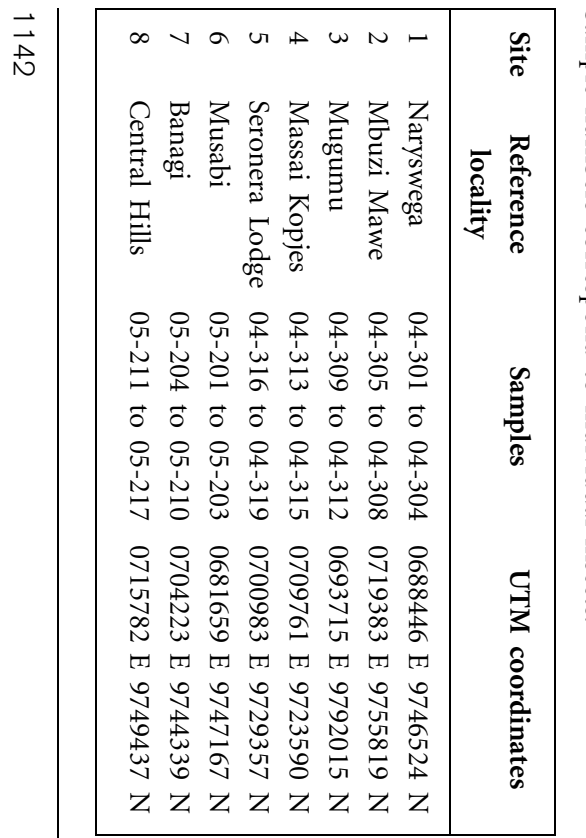

\begin{tabular}{|c|c|}
\hline 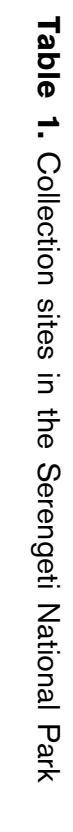 & 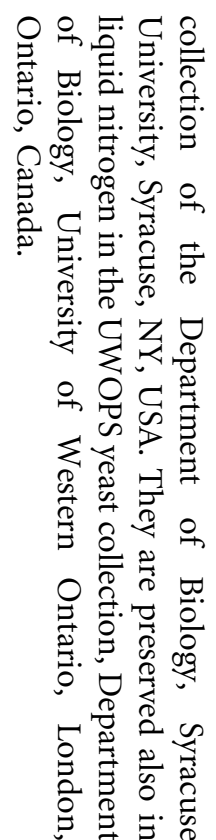 \\
\hline
\end{tabular}
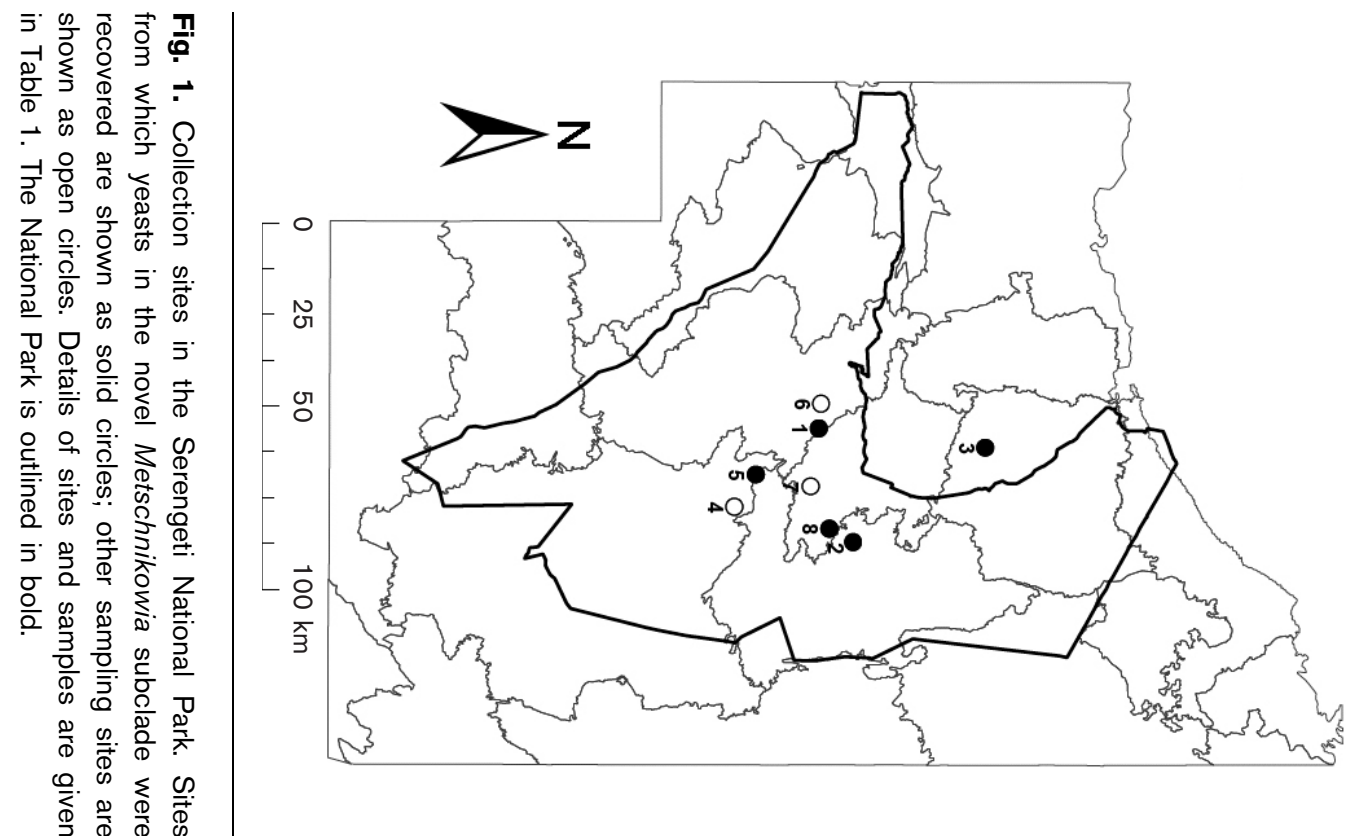

Table 2. Selected properties of Metschnikowia isolates from the Serengeti

Strain accession numbers are those in the yeast culture collection of the Department of Biology, Syracuse University, Syracuse, NY, USA (SUB), the Centraalbureau voor Schimmelcultures, Utrecht, The Netherlands (CBS), and the ARS-USDA Culture Collection, Peoria, IL, USA (NRRL). Mating reactions: sterile, asci; 1, single-spored asci; 2, two-spored asci. ${ }^{\mathrm{T}}$, Holotype; ${ }^{\mathrm{A}}$, allotype.

\begin{tabular}{|c|c|c|c|c|c|c|}
\hline Strain & $\begin{array}{c}\text { rDNA sequence } \\
\text { type }\end{array}$ & $\begin{array}{c}\text { Species } \\
\text { assignment }\end{array}$ & $\begin{array}{c}\text { Mating } \\
\text { type }\end{array}$ & $\begin{array}{l}\text { Mating with } \\
\text { SUB 05-213.1 }\end{array}$ & $\begin{array}{l}\text { Collection } \\
\text { site }\end{array}$ & Insect \\
\hline SUB $04-304.1$ & A & Metschnikowia sp. & $\mathrm{h}^{+}$ & Sterile, 1 & 1 & Mylabris tristigma \\
\hline SUB $04-306.1$ & A & Metschnikowia sp. & $\mathrm{h}^{+}$ & Sterile, 1 & 2 & Buprestid \\
\hline SUB $04-307.1(=$ CBS $10291=$ NRRL Y-27923 $)$ & B & Metschnikowia sp. & $\mathrm{h}^{+}$ & Sterile, 1,2 & 2 & Buprestid \\
\hline SUB $04-310.1(=$ CBS $10292=$ NRRL Y-27924 $)$ & $\mathrm{C}$ & Metschnikowia sp. & $\mathrm{h}^{+}$ & Sterile, 1 & 3 & Mylabris tristigma \\
\hline SUB $04-311.1$ & B & Metschnikowia sp. & $\mathrm{h}^{+}$ & Sterile, 1 & 3 & Mylabris tristigma \\
\hline SUB $04-318.1$ & B & Metschnikowia sp. & $\mathrm{h}^{+}$ & Sterile, 1 & 5 & Mylabris tristigma \\
\hline SUB $05-212.1$ & A & M. aberdeeniae & $\mathrm{h}^{-}$ & - & 8 & Scaptodrosophila sp. \\
\hline SUB $05-213.1^{\mathrm{T}}\left(=\mathrm{CBS} 10289^{\mathrm{T}}=\mathrm{NRRL} Y-27921^{\mathrm{T}}\right.$ & A & M. aberdeeniae & $\mathrm{h}^{-}$ & - & 8 & Mylabris tristigma \\
\hline SUB $05-213.2^{\mathrm{A}}(=\mathrm{CBS} 10290=\mathrm{NRRL} Y-27922)$ & A & M. aberdeeniae & $\mathrm{h}^{+}$ & 2 & 8 & Mylabris tristigma \\
\hline SUB $05-215.1$ & A & M. aberdeeniae & $\mathrm{h}^{+}$ & 2 & 8 & Buprestid \\
\hline SUB $05-217.1 \mathrm{a}$ & A & M. aberdeeniae & $\mathrm{h}^{+}$ & 2 & 8 & Scaptodrosophila sp. \\
\hline
\end{tabular}


Largely for reasons of logistics, a detailed quantitative survey of the yeast community of the flowers and their insects was not performed. However, Metschnikowia was clearly the dominant genus recovered in these collections. The ascomycetous yeasts Kodamaea kakaduensis and Candida restingae, both of which are typical of ephemeral flower insects (Lachance et al., 2001), were each recovered twice. Three single isolates were identified as Candida parapsilosis, a Candida glabrata-like species and an unknown Williopsis species. Other incidental yeasts included representatives of the genera Cryptococcus, Rhodotorula and other airborne fungi such as Aureobasidium, none of which was identified to the species level.

Two of the 11 isolates were of mating type $\mathrm{h}^{-}$. Assignment was based on the observation of conjugation tubes (not shown) in mixtures with the type $\left(\mathrm{h}^{+}\right)$of Metschnikowia continentalis (UFMG 96-173 ${ }^{\mathrm{T}}$ ). The tubes reached $40 \mu \mathrm{m}$ in length, but adhesion was not observed. None of the isolates, regardless of mating type, reacted in a similar manner when mixed with the allotype of $M$. continentalis $\left(\mathrm{h}^{-}\right.$, UFMG 96-179). Mating in all compatible pairs of African isolates gave rise to asci that varied in abundance and sporulation intensity. The five strains recovered from site 8 in 2005 (to be included in $M$. aberdeeniae) exhibited full interfertility, as demonstrated by the formation of abundant two-spored asci (Table 2, Fig. 2a-c); both $\mathrm{h}^{-}$strains were among these. Crosses between these and the remaining (2004) strains gave rise to fewer asci that were most frequently sterile (Fig. 2f) or single-spored (Fig. 2e, g). Rare two-spored asci (Fig. 2d) were detected in a cross with strain SUB 04-307.1. These interfertility patterns are not entirely consistent with the relatedness inferred from rDNA sequence divergence. The strains collected in 2005 had ITS-D1/D2 sequences identical to those of strains SUB 04-304.1 and SUB 04-306.1, in spite of their apparently lower cross-fertility. Three other strains differed by three substitutions (one in the ITS2 sequence and two in the D1 domain). Strain SUB 04-310.1 exhibited a high degree $(8 \cdot 6 \%)$ of sequence divergence from the rest. The differences (substitutions and indels) were spread across the ITS1, ITS2, D1 and D2 regions. The formation of single-spored asci in crosses involving that strain is therefore surprising.

The prudent conclusion at this time is that only the 2005 isolates should be assigned to a single reproduction-based species. The potential membership of the other strains, including those with identical sequences, will remain uncertain until additional $\mathrm{h}^{-}$isolates become available. The formation of abundant two-spored asci by every strain, when suitably crossed, would lend considerably more weight to additional species assignments. Although an excellent correlation is normally observed between biological species boundaries and the degree of divergence in rDNA sequences (Kurtzman \& Robnett, 1998), exceptions do exist. For example, some large-spored Metschnikowia species recently found in endemic beetles of Hawaii (Lachance et al., 2005) could not be delineated strictly on the basis of sequence

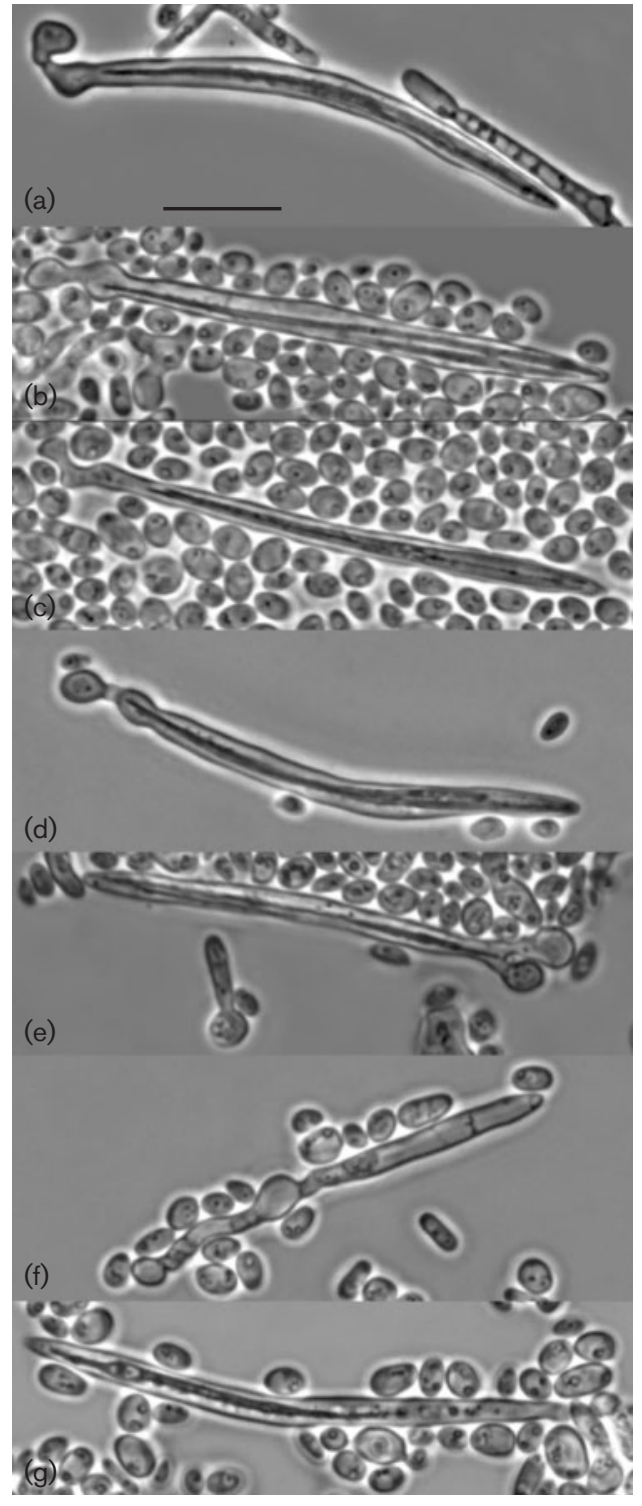

Fig. 2. Phase-contrast micrographs of asci of $M$. aberdeeniae strains and related isolates, resulting from crosses between strain SUB 05-213.1 ${ }^{\top}$ and strains SUB 05-213.2 (a, b), SUB 05-215.1 (c), SUB 04-307.1 (d), SUB 04-310.1 (e) and SUB 04-318.1 (f, g). Asci were two-spored (a-d), single-spored (e, g) or sterile (f). Bar, $10 \mu \mathrm{m}$.

divergence, although clear reproductive-compatibility groups were identified. The formation of sterile or single-spored asci should not be interpreted as a sign of conspecificity, as shown recently for Metschnikowia borealis and M. continentalis (Marinoni \& Lachance, 2004). Even the occasional formation of two-spored asci is possible in crosses between some sister species of Metschnikowia (Lachance \& Bowles, 2004). In both of these examples, correct species assignments required the verification of spore viability. In both cases also, it was found that spores that are formed in pairs in the majority of the asci arising in a cross are viable. 


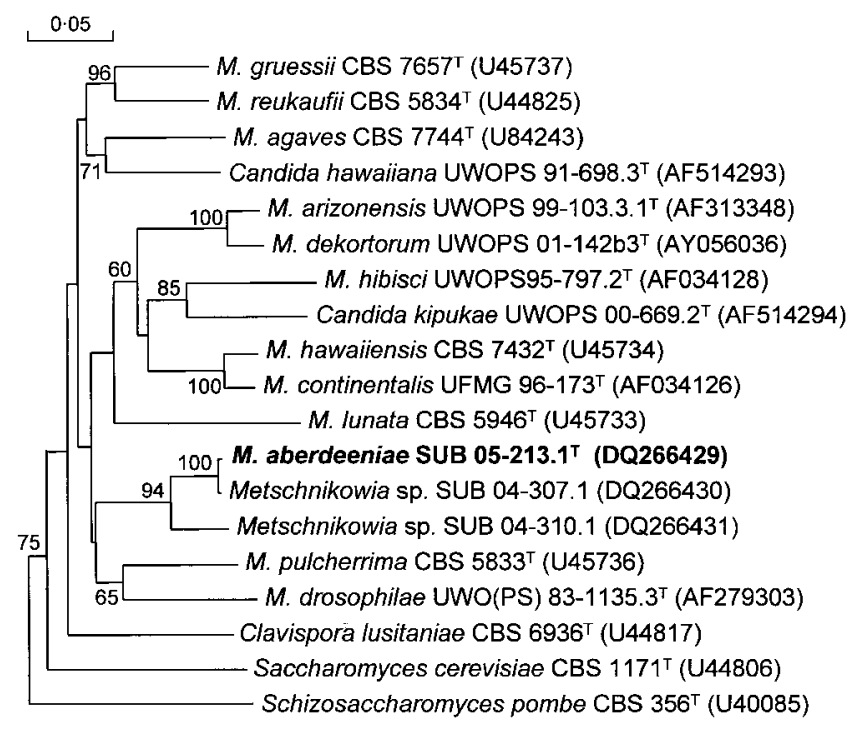

Fig. 3. Neighbour-joining phylogram of D1/D2 large-subunit rDNA sequences of selected species of the Metschnikowiaceae. The sequences were chosen so as to represent a broad range of subclades within the group. Bootstrap values of $50 \%$ and above are shown (1000 iterations). Branch lengths represent uncorrected distances. Bar, $5 \%$ sequence divergence.

The tree presented in Fig. 3 is based on an alignment of the D1/D2 variable domains of the large-subunit rDNA and illustrates the basal position of the African isolates in the clade. The other suggestion arising from the sequence data is that the diversity of flower beetle-associated Metschnikowia species in Africa may be just as extensive as that found in the New World or in Hawaii. This inference emerges from the observation that the sequence divergence among the Serengeti isolates exceeds that found among members of the core large-spored subclade (represented here by M. continentalis and Metschnikowia hawaiiensis) or the Metschnikowia arizonensis subclade (represented also by Metschnikowia dekortorum). The first subclade contains 10 described species and at least three undescribed species; the second subclade has four described species and two undescribed species.

The rDNA segment encompassing most of the SSU and most of the LSU was sequenced from strain SUB 05-213.1 ${ }^{\mathrm{T}}$ (GenBank accession no. DQ266429). Neither coding region supported a convincing placement of the species in one subclade or another in the Metschnikowiaceae. Whereas some regions in the small-subunit rDNA clearly suggested an affinity with the core large-spored group, others supported contradictory kinships. Potentially relevant to the basal status of the novel subclade is the fact that this is the first case in which the principal vectors of morning gloryassociated Metschnikowia species were not nitidulid beetles. This leaves unanswered the important question of whether the cladogenetic development of these yeasts follows biogeographical lines or alternatively embodies a history of co-speciation with vector insects. A continued search for nectar-feeding nitidulid beetles in African flowers might provide relevant clues in the future.

The cells and asci (Fig. 1) of M. aberdeeniae and relatives are typical of those of the core large-spored species, although the ascus size sits at the lower end of the known spectrum, not unlike that seen in Metschnikowia hamakuensis (Lachance et al., 2005). By contrast, the asci and ascospores of members of the $M$. arizonensis subclade (Lachance \& Bowles, 2002, 2004), which is a sister group to the core species, are more divergent morphologically. The formation of very long aseptate tubes, which is widespread in the core large-spored group, was not observed in $M$. aberdeeniae. Likewise, after a full month in Dalmau plate culture, only short chains of submerged cells were formed, to the exclusion of anything that could be construed as pseudohyphal growth, also common in both the largespored and the M. arizonensis clades.

The growth responses of the African isolates were typical of the majority of currently described Metschnikowia species, which in general are remarkably similar. In fact, it would be more or less impossible, on the basis of growth profiles alone, to discern the African isolates, not only from most large-spored species, but also from more distant relatives such as Metschnikowia reukaufii. Most of the isolates were homogeneous in terms of growth responses, except for some trivial variations in the rates of assimilation of a few carbon compounds such as ethanol, citric acid, gluconic acid and glucono- $\delta$-lactone. Strain SUB 04-310.1 grew more weakly on galactose, $\beta$-glucosides, mannitol, glucitol and D-glucosamine as carbon sources, and on lysine as a nitrogen source. Fermentation was also less vigorous in that strain. This sort of variation has been observed, on occasion, in some strains of Metschnikowia lochheadii.

\section{Latin diagnosis of Metschnikowia aberdeeniae Lachance et Starmer sp. nov.}

In medio YM post dies tres cellulae singulae, binae, aut in catenis brevis, globosae aut ovoidae $(2-3 \times 3-5 \mu \mathrm{m})$. Cultura in agaro malti post dies 14 convexa tumulosaque, glabra, candida et butyrosa. In agaro carbonis fundamento post dies 14 pseudomycelium nec mycelium verum non formatur. Post dies unus, cellulae stirpum interfertilium mixtarum in agaro carbonis fundamento tubi junctionis formantur. Post dies tres, magni asci cylindrati possunt videri. Asci stabiles sunt. Ascosporae aculeatae $(1 \cdot 1-1 \cdot 5 \times 35-50 \mu \mathrm{m})$. Glucosum et trehalosum (variabile et exigue) fermentantur. Glucosum, sucrosum, galactosum, trehalosum, maltosum, melezitosum, cellobiosum, salicinum, L-sorbosum, D-xylosum, ethanolum (lente et exigue), glycerolum (lente), ribitolum, xylitolum, mannitolum, glucitolum, acidum succinicum (aliquando lente), acidum gluconicum (exigue aut lente), glucono- $\delta$ lactonum (variabile), 2-ketogluconatum, glucosaminum (lente) et $N$-acetylglucosaminum, at non inulinum, raffinosum, melibiosum, lactosum, methyl $\alpha$-D-glucosidum, amylum solubile, L-rhamnosum, L-arabinosum, D-arabinosum, 
D-ribosum, methanolum, 1-propanolum, 2-propanolum, 1-butanolum, erythritolum, galactitolum, myo-inositolum, acidum lacticum, acidum citricum (aliquando exigue), acetonum, ethyl acetas nec hexadecanum. Ethylaminum, lysinum et cadaverinum assimilantur at non natrium nitricum nec natrium nitrosum. Ad crescentiam vitaminae externae necessariae sunt. Augmentum in $32^{\circ} \mathrm{C}$, at non $34^{\circ} \mathrm{C}$.

Habitat insectos e flores Ipomoea hildebrandtii in Hortus Patrius Serengeti, Tanzania. Typus SUB $05-213 \cdot 1^{\mathrm{T}}\left(\mathrm{h}^{-}\right)$. Allotypus SUB 04-213.2. In collectione zymotica Centraalbureau voor Schimmelcultures, Trajectum ad Rhenum, sub no. CBS $10289^{\mathrm{T}}$ et CBS 10290 depositae sunt.

\section{Description of Metschnikowia aberdeeniae Lachance et Starmer sp. nov.}

Metschnikowia aberdeeniae (a.ber.dee' ni.ae. N.L. gen. sing. fem. n. aberdeeniae of Aberdeen, in honour of Virginia Aberdeen, in grateful recognition of her contributions to yeast ecology).

On YM agar after 3 days at $25^{\circ} \mathrm{C}$, cells are spherical to ovoid, occur singly, in parent-bud pairs or in short chains, and measure $2-3 \times 3-5 \mu \mathrm{m}$. After 2 weeks, colonies are convex and umbonate, glossy, white and butyrous. In Dalmau plate culture on yeast carbon base agar after 2 weeks, pseudohyphae and true hyphae are not formed. After 1 month, compact chains of undifferentiated, mostly submerged, cells are produced at the edge of the exposed part of the streak culture. On yeast carbon base agar at $18^{\circ} \mathrm{C}$, mixtures of cells of complementary mating types give rise to zygotes and asci after $12-16 \mathrm{~h}$. After 3 days, mature asci containing two acicular ascospores $(1 \cdot 1-1 \cdot 5 \times 35-50 \mu \mathrm{m})$ are formed in a persistent ascus that retains conspicuous vestiges of the conjugated parent cells (Fig. 2a-c). Fermentation: in glucose, gas production begins after 2-3 days and a full tube of gas develops after 10 days. With regard to other assimilated sugars, only trehalose is fermented weakly in some strains. Glucose, sucrose, galactose, trehalose, maltose, melezitose, cellobiose, salicin, sorbose, xylose, ethanol (weak or slow), glycerol (slow), ribitol (slow), xylitol, mannitol, glucitol, succinic acid (sometimes slow), gluconic acid (weak to slow), glucono- $\delta$-lactone (variable), 2-ketogluconic acid, D-glucosamine and $\mathrm{N}$-acetyl-Dglucosamine are assimilated, but inulin, raffinose, melibiose, lactose, methyl $\alpha$-D-glucoside, starch, L-rhamnose, L-arabinose, D-arabinose, D-ribose, methanol, 1-propanol, 2-propanol, 1-butanol, erythritol, galactitol, inositol, lactic acid, citric acid (sometimes weak), acetone, ethyl acetate and n-hexadecane are not assimilated. Ethylamine, lysine and cadaverine are utilized as nitrogen sources, but sodium nitrate and nitrite are not. Negative for growth in the absence of vitamins. Positive for growth in the absence of amino acids. Positive for growth at $32{ }^{\circ} \mathrm{C}$ and negative for growth at $34{ }^{\circ} \mathrm{C}$; growth at $33^{\circ} \mathrm{C}$ is variable and weak. Negative for hydrolysis of gelatin and casein. Positive for hydrolysis of Tween 80 . Negative for acid production on chalk agar. Positive for growth in the presence of $10 \% \mathrm{NaCl}$; negative for growth at $15 \% \mathrm{NaCl}$. Growth in the presence of $50 \%$ glucose is slow. Negative for growth in the presence of $10 \mathrm{mg}$ cycloheximide $\mathrm{l}^{-1}$. Positive for growth in the presence of $75 \mathrm{mg}$ cetyltrimethylammonium bromide $1^{-1}$. Negative for starch production and the diazonium blue $\mathrm{B}$ reaction.

The habitat is insects associated with flowers of Ipomoea hildebrandtii in the Serengeti National Park, Tanzania. The type strain, strain SUB $05-213.1^{\mathrm{T}}\left(\mathrm{h}^{-}\right)$, was recovered from a specimen of the meloid beetle Mylabris tristigma collected in the Serengeti National Park. The designated allotype is strain SUB 05-213.2 $\left(\mathrm{h}^{+}\right)$, recovered from the same sample. These strains have been deposited in the collection of the Yeast Division of the Centraalbureau voor Schimmelcultures, Utrecht, The Netherlands (and the ARS-USDA Culture Collection, Peoria, IL, USA), as strains CBS $10289^{\mathrm{T}}$ $\left(=\right.$ NRRL Y-27921 $\left.{ }^{\mathrm{T}}\right)$ and CBS $10290(=$ NRRL Y-27922), respectively.

\section{Acknowledgements}

We acknowledge funding from the National Science Foundation (W. T. S.) and the Natural Science and Engineering Council of Canada (M.-A.L.). The cooperation of Mark Ritchie is gratefully acknowledged. Thanks are extended to Virginia Aberdeen, Diana Lawrie and Jessica Dobson for laboratory assistance.

\section{References}

Abate, T. \& Ampofo, J. K. O. (1996). Insect pests of beans in Africa: their ecology and management. Annu Rev Entomol 41, 45-73.

Kurtzman, C. P. \& Robnett, C. J. (1998). Identification and phylogeny of ascomycetous yeasts from analysis of nuclear large subunit (26S) ribosomal DNA partial sequences. Antonie van Leeuwenhoek 73, 331-371.

Lachance, M. A. \& Bowles, J. M. (2002). Metschnikowia arizonensis and Metschnikowia dekortorum, two new large-spored yeast species associated with floricolous beetles. FEMS Yeast Res 2, 81-86.

Lachance, M. A. \& Bowles, J. M. (2004). Metschnikowia similis sp. nov. and Metschnikowia colocasiae sp. nov., two ascomycetous yeasts isolated from Conotelus spp. (Coleoptera: Nitidulidae) in Costa Rica. Stud Mycol 50, 69-76.

Lachance, M. A., Starmer, W. T., Rosa, C. A., Bowles, J. M., Barker, J. S. F. \& Janzen, D. H. (2001). Biogeography of the yeasts of ephemeral flowers and their insects. FEMS Yeast Res 1, 1-8.

Lachance, M. A., Ewing, C. P., Bowles, J. M. \& Starmer, W. T. (2005). Metschnikowia hamakuensis sp. nov., Metschnikowia kamakouana sp. nov., and Metschnikowia mauinuiana sp. nov., three endemic yeasts from Hawaiian nitidulid beetles. Int J Syst Evol Microbiol 55, 1369-1377.

Marinoni, G. \& Lachance, M. A. (2004). Speciation in the largespored Metschnikowia clade and establishment of a new species, Metschnikowia borealis comb. nov. FEMS Yeast Res 4, 587-596.

Yarrow, D. (1998). Methods for the isolation and identification of yeasts. In The Yeasts, a Taxonomic Study, 4th edn, pp. 77-100. Edited by C. P. Kurtzman \& J. W. Fell. Amsterdam: Elsevier. 